Pacific Journal of Mathematic 


\section{THE ABSOLUTE CONTINUITY OF TOEPLITZ'S MATRICES}

\section{MARVIN ROSENBLUM}

1. Introduction. Suppose $W$ is a real $L^{2}(-\pi, \pi)$ function that is bounded below but not equivalent to a constant function. The Toeplitz matrix associated with $W$ is $\boldsymbol{T}_{0}=\left[w_{j-k}\right], j, k=0,1,2, \cdots$, where

$$
w_{n}=\frac{1}{2 \pi} \int_{-\pi}^{\pi} W(\phi) e^{-i n \phi} d \phi, n=0, \pm 1, \pm 2, \cdots
$$

The hermitian matrix $\boldsymbol{T}_{0}$ gives rise to a semi-bounded transformation $T_{1}$ on complex sequential Hilbert space $l_{2}$, and thus the Friedrichs extension $\boldsymbol{T}$ of $\boldsymbol{T}_{1}$ is a self-adjoint operator. $\quad \boldsymbol{T}=\boldsymbol{T}(W(\phi))$ is the Toeplitz operator associated with $W$.

In [5], [6] Hartman and Wintner show that the case in which $W$ is not semi-bounded (which we prudently avoid here) presents special difficulty. However for semi-bounded $W$ they prove that

(i) the spectrum of $\boldsymbol{T}$ fills the interval

[ess inf $W$, ess sup $W$ ],

and

(ii) $\boldsymbol{T}$ has no point spectrum.

Thus the spectral measure ([4], p. 58) $\boldsymbol{E}(\cdot)$ of $\boldsymbol{T}$ is such that $\langle\boldsymbol{E}(\cdot) F, F\rangle$ is a nonatomic Borel measure for each $F \in l^{2}$. If $\langle\boldsymbol{E}(\cdot) F, F\rangle$ is $\mathrm{AC}$ (absolutely continuous with respect to Lebesgue measure) for each $F \in l^{2}$, then we say that $\boldsymbol{T}$ is $\mathrm{AC}$.

Our investigation continues work of C. R. Putnam [11]. He proves that $\boldsymbol{T}$ is AC in each of the following cases:

(i) $W(\phi)=2 \cos n \phi, n=1,2, \cdots$

(ii) $W(\phi)=2 \sin n \phi, n=1,2, \cdots$

(iii) Let $a_{j k}=w_{k-j}$ for $k-j \geqq 1$ and $a_{j k}=0$ otherwise.

Further suppose that the $\left\{w_{n}\right\}$ are real, that $\boldsymbol{A}_{0}=\left[a_{j k}\right]$ is bounded, and that 0 is not an eigenvalue of the Hankel matrix $\left[w_{j+k+1}\right], j, k=0,1,2, \cdots$.

For case (i) Putnam gives a more complete spectral analysis. $\mathrm{He}$ applies the perturbation theory propounded in [13] to prove the following result:

$1.2 T(2 \cos n \phi)$ is unitarily equivalent to $2 T_{n}\left(\frac{1}{2} T(2 \cos \phi)\right)$. Here $T_{n}$ is the $n$th degree Tchebichef polynomial, $n=1,2, \cdots$.

Received July 20, 1959. This research was supported by the United States Air Force through the Air Force Office of Scientific Research of the Air Research and Development Command, under contract AF 49 (638)-72 at the University of Virginia. 
In $\S \S 2$ and 3 we prove that every Toeplitz operator is AC. The method of proof first involves deriving a generating function formula for the resolvent of $\boldsymbol{T}$. This formula appears in the work [2] of Calderon, Spitzer, and Widom. However, we shall offer a different derivation, one that points out an interesting connection between $\boldsymbol{T}$ and the Szego kernel function. Next we shall apply a result from the AronszajnDonoghue [1] theory of exponential representations of analytic functions, and consequently deduce that $\boldsymbol{T}$ is absolutely continuous. We conclude with $\S 4$ where 1.2 is generalized. We elaborate on Putnam's method. By severely restricting $W$ we are able to employ Kato's generalization [7], [8] of [13] to exhibit a multiplication operator $\boldsymbol{M}_{\mathrm{AC}}$ on an $L^{2}$ space such that $T$ is unitarily equivalent to $\boldsymbol{M}_{\mathrm{AC}}$.

2. $\boldsymbol{T}$ and the Szegö kernel function. We first set down some notation. We shall ambiguously employ " $F$ " to denote

(a) the element $\left\{f_{n}\right\}_{0}^{\infty}$ of $l^{2}$;

(b) the element $F\left(e^{i \phi}\right)$ of $L^{2}(-\pi, \pi)$ that has the Fourier series $\sum_{n=0}^{\infty} f_{n} e^{i n \phi} ;$ and

(c) the holomorphic function $F(u)=\sum_{n=0}^{\infty} f_{n} u^{n},|u|<1$.

Let $\langle$,$\rangle be the l^{2}$ inner product and suppose $*$ is the symbol of complex conjugation, used so $F^{*}\left(e^{i \phi}\right) \sim \sum_{n=0}^{\infty} f_{n}^{*} e^{i n \phi}$ and $\left[F\left(e^{i \phi}\right)\right]^{*} \sim \sum_{n=0}^{\infty}$ $f_{n}^{*} e^{-i n \phi}$. Then

$$
\langle F, G\rangle=\sum_{n=0}^{\infty} f_{n} g_{n}^{*}=\frac{1}{2 \pi} \int_{-\pi}^{\pi} F\left(e^{i \phi}\right)\left[G\left(e^{i \phi}\right)\right]^{*} d \phi .
$$

We suppose that $u, v$ are complex numbers such that $|u|<1,|v|<1$, and define $U=\left\{u^{n}\right\}_{0}^{\infty} \in l^{2}, V=\left\{v^{n}\right\}_{0}^{\infty} \in l^{2}$. Note that $U\left(e^{i \phi}\right)=\left(1-u e^{i \phi}\right)^{-1}$ and $V^{*}\left(e^{i \phi}\right)=\left(1-v^{*} e^{i \phi}\right)^{-1}$.

Select $\lambda$ so that $1+\lambda \leqq$ ess inf $W$. Let $l^{2, \lambda}$ be the inner product space formd of elements $F \in l^{2}$ such that

$$
[F, F]=\frac{1}{2 \pi} \int_{-\pi}^{\pi}\left|F\left(e^{i \phi}\right)\right|^{2}(W(\phi)-\lambda) d \phi<\infty .
$$

Since

$$
[F, F] \geqq\langle F, F\rangle
$$

it follows that $l^{2 \lambda}$ is a (complete) Hilbert space. Define the linear functional $L_{v}$ on $l^{2, \lambda}$ by $L_{v}(F)=\left\langle F, V^{*}\right\rangle . L_{v}$ is bounded since

$$
\left|L_{v}(F)\right|^{2} \leqq\langle F, F\rangle\left\langle V^{*}, V^{*}\right\rangle \leqq[F, F]\left\langle V^{*}, V^{*}\right\rangle \text {. }
$$

Hence by the Frechet-Riesz representation theorem ([12], p. 61) there exists a unique element $K_{v} \in l^{2, \lambda}$ such that $\left[F, K_{v}\right]=L_{v}(F)$. Thus

$$
F(v)=\left\langle F, V^{*}\right\rangle=\left[F, K_{v}\right]
$$




$$
=\frac{1}{2 \pi} \int_{-\pi}^{\pi} F\left(e^{i \phi}\right)\left[K_{v}\left(e^{i \phi}\right)\right]^{*}(W(\phi)-\lambda) d \phi
$$

$$
\text { for all } v,|v|<1 \text {. }
$$

It follows from 2.2 that $K_{v}(u)=\left\langle K_{v}, U^{*}\right\rangle$ is the Szegö kernel function associated with the Hilbert space of holomorphic functions $F$ such that $[F, F]$ is finite. From ([3], p. 51);

2.3

$$
K_{v}(u)=\left(1-u v^{*}\right)^{-1}[g(v)]^{*} g(u),
$$

where

$2.4 \quad g(u)=\exp -\frac{1}{4 \pi} \int_{-\pi}^{\pi} \log (W(\phi)-\lambda)\left(e^{i \phi}+u\right)\left(e^{i \phi}-u\right)^{-1} d \phi$.

We next turn our attention to the Toeplitz matrix $\boldsymbol{T}_{0}$. We define the transformation $\boldsymbol{T}_{1}$ to be the restriction of $\boldsymbol{T}_{0}$ to the subset $\mathscr{D}_{1}$ of $l^{2}$ consisting of elements $F$ that have only a finite number of non-zero components. Then if $F \in \mathscr{D}_{1}$, and $\delta$ is the Kronecker symbol,

$$
\langle(T-\lambda) F, F\rangle=\sum\left(w_{k-j}-\lambda \delta_{j, k}\right) f_{j} f_{k}^{*}=[F, F] \text {. }
$$

Since $[F, F] \geqq\langle F, F\rangle$ we are in a situation to which the Friedrichs extension theory is applicable ([12], p. 328-333). Upon applying this theory we note that:

(a) There exists a unique self-adjoint operator $\boldsymbol{T}$ that is an extension of $T_{1}$ and whose domain $\mathscr{D}$ is contained in $l^{2, \lambda} . \mathscr{D}$ is a independent of the choice of $\lambda+1 \leqq$ ess inf $W$. Notice that $\boldsymbol{T}$ is a quite convenient self-adjoint extension of $\boldsymbol{T}_{1}$ since it preserves the analytic nicety 2.5 for all $F \in \mathscr{D}$.

(b) $(\boldsymbol{T}-\lambda)^{-1}$ is a bounded positive definite operator that maps $l^{2}$ into $l^{2, \lambda}$, and furthermore

$$
\langle F, G\rangle=\left[F,(T-\lambda)^{-1} G\right] \text { for all } G \in l^{2} \text { and } F \in l^{2, \lambda} .
$$

Theorem 1. Suppose $\lambda+1 \leqq$ ess inf $W$. Then $(\boldsymbol{T}-\lambda)^{-1}$ exists, is bounded, and $\left\langle(\boldsymbol{T}-\lambda)^{-1} V^{*}, U^{*}\right\rangle=K_{v}(u)$.

Proof. Suppose $F \in l^{2, \lambda}$. Then by 2.1 and 2.2, $\left\langle F, V^{*}\right\rangle=\sum_{n=0}^{\infty}$ $f_{n} v^{n}=F(v)=\left[F, K_{v}\right]$. But, by 2.6, $\left\langle F, V^{*}\right\rangle=\left[F,(\boldsymbol{T}-\lambda)^{-1} V^{*}\right]$. Thus $K_{v}=(\boldsymbol{T}-\lambda)^{-1} V^{*}$, and $K_{v}(u)=\left\langle K_{v}, U^{*}\right\rangle=\left\langle(\boldsymbol{T}-\lambda)^{-1} V^{*}, U^{*}\right\rangle$, as asserted.

As commented before, Theorem 1 can be derived from results in Calderon, Spitzer, and Widom's paper [2].

3. Exponential representation. We list some of the results of the Aronszajn-Donoghue theory of exponential representations of holomorphic functions in

THEOREM 2. Suppose $R$ is a function holomorphic in the upper 
half plane and there having a non-negative imaginary part. Then:

(i) ([1], p. 325). There exists a positive measure $\mu$ and real numbers $\alpha^{\prime} \geqq 0$ and $\beta$ such that

$$
R(\lambda)=\alpha^{\prime} \lambda+\beta+\int_{-\infty}^{\infty}\left[(t-\lambda)^{-1}-t\left(t^{2}+1\right)^{-1}\right] d \mu .
$$

$\alpha^{\prime} \beta, \mu$ are uniquely determined by $R$, and $\left(t^{2}+1\right)^{-1}$ is integrable with respect to $\mu$. If $|t|\left(t^{2}+1\right)^{-1}$ is integrable with respect to $\mu$, then

$$
\begin{gathered}
R(\lambda)=\alpha^{\prime} \lambda+\beta^{\prime}+\int_{-\infty}^{\infty}(t-\lambda)^{-1} d \mu, \text { where } \\
\beta^{\prime}=\beta-\int_{-\infty}^{\infty} t\left(t^{2}+1\right)^{-1} d \mu
\end{gathered}
$$

(ii) ([1], p. 331). There exists a Lebesgue measurable function $\alpha$ with $0 \leqq \alpha \leqq 1$ and a real number $\sigma$ such that

$$
R(\lambda)=\exp \sigma \exp \int_{-\infty}^{\infty}\left[(t-\lambda)^{-1}-t\left(t^{2}+1\right)^{-1}\right] \alpha(t) d t
$$

$\alpha$ is determined by 3.3 modulo a set of Lebesgue measure zero.

(iii) ([1], p. 386). A sufficient condition for $\mu$ to be $\mathrm{AC}$ is that for all real $x$

3.4

$$
\omega(x)=\lim \{\omega(a, b): a \uparrow x, b \downarrow x\}<1,
$$

where

$$
\omega(\alpha, b)=\sup \{\alpha(d)-\alpha(c): a<c<d<b\} .
$$

We next reframe 2.3 in a form suitable for application of the preceding theorem. Let $\chi_{t}$ be the characteristic function of $\{\phi: W(\phi) \leqq t$, $-\pi<\phi \leqq \pi\}$. Put

$$
P(\phi, u, v)=\frac{1}{4 \pi}\left[\left(e^{i \phi}+u\right)\left(e^{i \phi}-u\right)^{-1}+\left(e^{-i \phi}+v^{*}\right)\left(e^{-i \phi}-v^{*}\right)^{-1}\right],
$$

so if

$$
v=r e^{i \psi}, P(\phi, v, v)=\frac{1}{2 \pi}\left(1-r^{2}\right)\left(1-2 r \cos (\phi-\psi)+r^{2}\right)^{-1}
$$

is the Poisson kernel. Let

$$
\sigma(u, v)=-\frac{1}{2} \int_{-\pi}^{\pi} \log \left[1+(W(\phi))^{2}\right] P(\phi, u, v) d \phi,
$$

and $\alpha(t, u, v)=\int_{-\pi}^{\pi} \chi_{t}(\phi) P(\phi, u, v) d \phi$. Notice that $\alpha(\cdot, u, v)$ is of bounded variation, with $\alpha(t, u, v)=0$ or 1 according to whether $t<\operatorname{essinf}$ $W$ or $t>$ ess sup $W$ respectively. Also note that $\alpha(\cdot, v, v)$ is monotone increasing with $0 \leqq \alpha(t, v, v) \leqq 1$.

Lemma 1. If $\Im_{\mathfrak{m}} \lambda \neq 0$ or $\lambda<\operatorname{ess}$ inf $W$, then 


$$
\begin{aligned}
& \left(1-u v^{*}\right)\left\langle(\boldsymbol{T}-\lambda)^{-1} V^{*}, U^{*}\right\rangle \\
& \quad=\exp \sigma(u, v) \exp \int_{-\infty}^{\infty}\left[(t-\lambda)^{-1}-t\left(t^{2}+1\right)^{-1}\right] \alpha(t, u, v) d t
\end{aligned}
$$

Proof. Temporarily assume that

$\left.{ }^{*}\right) \lambda+1 \leqq$ ess inf $W$. By 2.3 and Theorem 1

$$
\begin{aligned}
& \left(1-u v^{*}\right)\left\langle(\boldsymbol{T}-\lambda)^{-1} V^{*}, U^{*}\right\rangle=\exp -\int_{-\pi}^{\pi} \log (W(\phi)-\lambda) P(\phi, u, v) d \phi \\
& =\exp \sigma(u, v) \exp -\int_{-\pi}^{\pi} \log \left[(W(\phi)-\lambda)\left((W(\phi))^{2}+1\right)^{-1 / 2}\right] P(\phi, u, v) d \phi \\
& =\exp \sigma(u, v) \exp -\int_{-\infty}^{\infty} \log \left[(t-\lambda)\left(t^{2}+1\right)^{-1 / 2}\right] d_{t} \alpha(t, u, v) .
\end{aligned}
$$

We integrate by parts to obtain 3.5 under assumption $\left(^{*}\right)$. An analytic continuation argument enables us to relax $(*)$.

We now apply Theorem 2.

Lemma 2. Suppose $|v|<1$. Then $\left\langle\boldsymbol{E}(\cdot) V^{*}, V^{*}\right\rangle$ is AC.

Proof. Consider $R(\lambda)=\left(1-|v|^{2}\right)\left\langle(\boldsymbol{T}-\lambda)^{-1} V^{*}, V^{*}\right\rangle$. This is a holomorphic function of the type described in Theorem 2. 3.5 assures us that it has the exponential representation 3.3 with $\alpha(t)=\alpha(t, v, v)$. We shall show that $\alpha$ satisfies 3.4 and from this it will follow that $\mu(\cdot)=\left\langle\boldsymbol{E}(\cdot) V^{*}, V^{*}\right\rangle$ is AC. Now,

$$
\begin{aligned}
\omega(a, b) & =\sup \left\{\int_{-\pi}^{\pi}\left[\chi_{d}(\phi)-\chi_{c}(\phi)\right] P(\phi, v, v) d \phi: a<c<d<b\right\} \\
& \leqq \int_{-\pi}^{\pi}\left[\chi_{b-}(\phi)-\chi_{a+}(\phi)\right] P(\phi, v, v) d \phi
\end{aligned}
$$

since $P(\cdot, v, v)$ is positive. Thus

$$
\omega(x) \leqq \int_{-\pi}^{\pi}\left[\chi_{x+}(\phi)-\chi_{x-}(\phi)\right] P(\phi, v, v) d \phi=h(r, \psi), \quad \text { where } v=r e^{i \psi r} .
$$

Since $P(\phi, v, v)$ is the Poisson kernel, $h$ is a non-negative harmonic function in $|v|<1$. $W$ is not equivalent to a constant, so $h$ is not a constant function. Thus by the maximum principle, $h(r, \psi)<1$ if $r<1$. We invoke 3.4 to complete the proof.

Now we can settle

\section{Theorem 3. $\boldsymbol{T}$ is AC.}

Proof. From now on let $\nu$ be real Lebesgue measure as restricted to the real Borel sets $\mathscr{B}$. Assume $\nu(\Delta)=0$. Lemma 2 assures us that if $|v|<1$. then $\left\langle\boldsymbol{E}(\Delta) V^{*}, V^{*}\right\rangle=0$. Suppose now that $F \in l^{2}$. We use the Schwarz inequality and the fact that $\boldsymbol{E}(\Delta)$ is a projection to 
note that

$$
\begin{aligned}
\left|\left\langle\boldsymbol{E}(\Delta) V^{*}, F\right\rangle\right| \leqq\left\|\boldsymbol{E}(\Delta) V^{*}\right\|\|\boldsymbol{F}\| & =\left[\left\langle\boldsymbol{E}(\Delta) V^{*}, \boldsymbol{E}(\Delta) V^{*}\right\rangle\right]^{1 / 2}\|F\| \\
& =\left[\left\langle\boldsymbol{E}(\Delta) V^{*}, V^{*}\right\rangle\right]^{1 / 2}\|F\|=0 .
\end{aligned}
$$

Thus $\left\langle\boldsymbol{E}(\Delta) V^{*}, F\right\rangle=0$ for all $v,|v|<1$. But the set $\left\{V^{*}:|v|<1\right\}$ is fundamental in $l^{2}$ since $\left\langle G, V^{*}\right\rangle=\sum_{n=0}^{\infty} g_{n} v^{n}=0$ for all $v,|v|<1$ implies that the $g_{n}$ all vanish. Thus $\langle\boldsymbol{E}(\Delta) F, F\rangle=0$, and $\boldsymbol{T}$ is AC.

4. Spectral theory. Our principal goal now is to establish a spectral analysis for $\boldsymbol{T}$. More particularly, we wish to exhibit a multiplication operator $\boldsymbol{M}_{\mathrm{AC}}$ on an $L^{2}$ space such that $\boldsymbol{M}_{\mathrm{AC}}$ is unitarily equivalent to $\boldsymbol{T}$. However, we were able to achieve this goal only for a small class of $\boldsymbol{T}(W(\phi))$. From now on we assume that $W$ is even and AC, and that the derivative $W^{\prime}$ of $W$ has an absolutely convergent Fourier series, so $\sum_{n}\left|w_{n}\right|<\infty$. Our techniques follow those of Putnam [11], but whereas he uses the theory presented by this author in [13], we use T. Kato's generalization [7], [8] of [13]. See also [9] and [10].

We start by discussing some preliminary material that we include here for completeness. A countably-additive function $\boldsymbol{E}$ on $\mathscr{P}$ to projection operators in a Hilbert space $\mathscr{L}$ is $\mathrm{AC}$ if $\nu(\Delta)=0$ implies $\boldsymbol{E}(\Delta)=0$. $\boldsymbol{E}$ is singular if there exists $\beta \in \mathscr{B}$ such that $\nu(\beta)=0$ but $E(\Delta \cap \beta)$ $=E(\Delta)$ for all $\Delta \in \mathscr{B}$. It is easy to see that a self-adjoint operator $\boldsymbol{M}$ is $\mathrm{AC}$ if and only if its spectral measure is AC.

We shall now establish a Lebesgue decomposition theorem for spectral measures as a corollary of the classical version of that theorem.

Lemma 3. Suppose $\boldsymbol{E}(\cdot)$ is a spectral measure in a separable Hilbert space $\mathscr{L}$. Then:

(i) There exists $\gamma \in \mathscr{B}$ with $\nu(-\gamma)=0$ and such that

$$
\boldsymbol{E}_{A O}(\cdot)=\boldsymbol{E}(\cdot \cap \gamma) \text { is an } \mathrm{AC} \text { and }
$$

$$
\boldsymbol{E}_{S}(\cdot)=\boldsymbol{E}(\cdot-\gamma) \text { is a singular projection-valued measure. }
$$

(ii) If $F, G \in \mathscr{L}, \Delta \in \mathscr{B}$, and $\boldsymbol{E}$. is the resolution of the identity associated with $\boldsymbol{E}(\cdot)$, then

$$
\left\langle\boldsymbol{E}_{\Lambda C}(\Delta) F, G\right\rangle=\int_{\Delta} d\left\langle\boldsymbol{E}_{x} F, G\right\rangle \mid d x d \nu .
$$

(iii) The decomposition $\boldsymbol{E}(\cdot)=\boldsymbol{E}_{\mathrm{AC}}(\cdot)+\boldsymbol{E}_{s}(\cdot)$ of $\boldsymbol{E}(\cdot)$ as the sum of an $\mathrm{AC}$ and singular measure is unique.

Proof. Suppose $F, G \in \mathscr{L}, \Delta \in \mathscr{B}$. Then since $\langle\boldsymbol{E} . F, G\rangle$ is of bounded variation it has a a derivative a.e. that is $\nu$-summable. Also

$$
\int_{4} d\left\langle\boldsymbol{E}_{x} F, F\right\rangle \mid d x d \nu \leqq \int_{4} d\langle\boldsymbol{E}(\cdot) F, F\rangle=\leqq \boldsymbol{E}(\Delta) F, F \leqq\|F\|^{2},
$$


so the first term above represents a bounded quadratic form. Thus by ([4], p. 33), $b(F, G)=\int_{\Delta} d\left\langle\boldsymbol{E}_{x} F, G\right\rangle \mid d x d \nu$ is a bounded bilinear functional, so there exists a bounded operator $\boldsymbol{E}_{\mathrm{AC}}(\Delta)$ such that $\left\langle\boldsymbol{E}_{\mathrm{AC}}(\Delta) F\right.$, $G\rangle=b(F, G)$ for all $F, G . \quad \boldsymbol{E}_{\mathrm{AC}}(\cdot)$ is clearly countably additive on $\mathscr{B}$, and thus so is $\boldsymbol{E}_{S}(\cdot)=\boldsymbol{E}(\cdot)-\boldsymbol{E}_{\mathrm{AC}}(\cdot)$.

Let $\left\{F_{j}\right\}_{j=0}^{\infty}$ be a countable dense subset of $\mathscr{L}$. By the classical version of the Lebesgue decomposition theorem as found in ([14], p. 119), corresponding to each pair $j, k$ of non-negative integers there exists $\beta_{j, k} \in B$ such that $\nu\left(\beta_{j, k}\right)=0$ and

$$
\left\langle\boldsymbol{E}(\Delta) F_{j}, F_{k}\right\rangle=\left\langle\boldsymbol{E}\left(\Delta \cap \beta_{j, k}\right) F_{j}, F_{k}\right\rangle+\left\langle\boldsymbol{E}_{\mathrm{AC}}(\Delta) F_{j}, F_{k}\right\rangle
$$

for all $\Delta \in \mathscr{B}$. Let $\beta$ be the union of all the $\beta_{j, k}, j, k=0,1,2, \cdots$. Then $\nu(\beta)=0$ and $\left(^{*}\right)$ holds with $\beta_{j, k}$ replaced by $\beta$. Now we pass from the dense subset to all of $\mathscr{L}$. For all $F, G \in \mathscr{L}, \Delta \in \mathscr{B}$

$$
\langle\boldsymbol{E}(\Delta) F, G\rangle=\langle\boldsymbol{E}(\Delta \cap \beta) F, G\rangle+\left\langle\boldsymbol{E}_{\mathrm{aC}}(\Delta) F, G\right\rangle,
$$

where the decomposition of the left hand term into singular and $\mathrm{AC}$ parts is unique. Put $\gamma=-\beta$. Then 4.2 holds and thus 4.1 is also true. (iii) follows from $(* *)$.

It follows from lemma 3 that $\boldsymbol{E}_{\mathrm{AC}}(\cdot)=\boldsymbol{E}(\gamma) \boldsymbol{E}(\cdot) \boldsymbol{E}(\gamma)$ is a spectral measure in the Hilbert space $\boldsymbol{E}(\gamma) \mathscr{L} . \boldsymbol{M}_{\mathrm{AC}}=\boldsymbol{E}(\gamma) \boldsymbol{M} \boldsymbol{E}(\gamma)$ is the self-adjoint operator on $\boldsymbol{E}(\gamma) \mathscr{L}$ having this spectral measure. $\boldsymbol{M}_{\mathrm{AC}}$ is obviously AC.

The following simple example will play a role in what happens later. Let $W$ be as before, even, with $\sum_{n}\left|w_{n}\right|<\infty$. Let $\boldsymbol{M}$ be the multiplication operator that maps any $F \in L^{2}(0, \pi)=\mathscr{L}$ into $W \cdot F \in \mathscr{L}$. Let $\chi(\Delta)$ be the characteristic function of $\{\phi: W(\phi) \in \Delta: 0 \leq \phi \leq \pi\}$. Since

$$
\langle\boldsymbol{M} F, F\rangle=\frac{1}{\pi} \int_{0}^{\pi} W(\phi)|F(\phi)|^{2} d \phi=\int_{-\infty}^{\infty} t d_{t} \frac{1}{\pi} \int_{0}^{\pi} \chi(\Delta)(\phi)|F(\phi)|^{2} d \phi
$$

it follows that the spectral measure $\boldsymbol{E}(\cdot)$ of $\boldsymbol{M}$ is defined by $\boldsymbol{E}(\Delta) F=$ $\chi(\Delta) \cdot F$. Lemma 3 guarantees the existence of $\gamma \in \mathscr{B}$ such the $1 / \pi$ $\int_{0}^{\pi} \chi(\gamma) \chi(\cdot)(\phi)|F(\phi)|^{2} d \phi$ is $\mathrm{AC}$ for all $F \in \mathscr{L}$, while $\boldsymbol{E}(\cdot-\gamma)$ is singular. $\boldsymbol{E}(\gamma) \mathscr{L}$ can be identified with the Hilbert space $L^{2}(A)$, where $F \in L^{2}(A)$ if and only if $\|F\|_{A}<\infty$, where

$$
\|F\|_{A}=\left[\frac{1}{\pi} \int_{0}^{\pi} \chi(\gamma)|F(\phi)|^{2} d \phi\right]^{1 / 2}=\left[\frac{1}{\pi} \int_{A}|F(\phi)|^{2} d \phi\right]^{1 / 2},
$$

and

$$
A=\{\phi: W(\phi) \in \gamma, \quad 0 \leqq \phi \leqq \pi\} .
$$

Similarly $\boldsymbol{M}_{\mathrm{AC}}$ can be considered to be the mapping that takes any $F \in L^{2}(A)$ into $W \cdot F \in L^{2}(A)$.

Another concept that we shall have cause to use is that of trace class. As is usual, a bounded operator on $l^{2}$ is identified with its 
matrix representation. A matrix $\boldsymbol{H}=\left[w_{j, k}\right], i, j=0,1,2, \cdots$ belongs to the Schmidt-Hilbert class SH if $\sum_{j, k=0}^{\infty}\left|w_{j, k}\right|^{2}<\infty . \quad \boldsymbol{H}$ belongs to the trace class TC if $\boldsymbol{H} \in \mathrm{SH}$ and $\|\boldsymbol{H}\|_{1}<\infty$, where $\|\boldsymbol{H}\|_{1}$ is the sum of the absolute values of the eigenvalues of $\boldsymbol{H}$ repeated according to multiplicity.

As an example we treat the Hankel matrix $\boldsymbol{H}=\left[w_{j+k+2}\right]$. As proved in [5], $\boldsymbol{H} \in \mathrm{SH}$ if and only if $\sum_{n=1}^{\infty} n\left|w_{n+1}\right|^{2}<\infty$. This follows from the equality $\sum_{j, k=0}^{\infty}\left|w_{j+k+2}\right|^{2}=\sum_{n=1}^{\infty} n\left|w_{n+1}\right|^{2}$, and gives a necessary condition that $\boldsymbol{H} \in \mathrm{TC}$. Now, define $\boldsymbol{H}_{n}=\left[\delta_{j+k+2, n}\right]$. Then $\boldsymbol{H}=\sum_{n=2}^{\infty}$ $w_{n} \boldsymbol{H}_{n}$. Since $\left\|\boldsymbol{H}_{n}\right\|_{1} \leqq n$ it follows that $\|\boldsymbol{H}\|_{1} \leqq \sum_{n=2}^{\infty}\left|w_{n}\right||| \boldsymbol{H}_{n} \|_{1} \leqq \sum_{n=2}^{\infty} n\left|w_{n}\right|$. Thus a sufficient condition $\boldsymbol{H} \in \mathrm{TC}$ is that $W$ be AC such that $W^{\prime}$ has an absolutely convergent Fourier series. This, of course, is part of our standing hypothesis on $W$ for this section. We do not know a useful necessary and sufficient condition for a Hankel matrix to belong to TC.

Hankel matrices enter into our picture, following an idea of Putnam's, via the following

Lemma 4. Let $\boldsymbol{H}$ be as as in the above example. Let $\boldsymbol{S}=\left[s_{j, k}\right]$, where $s_{j, k}=2 / \pi \int_{0}^{\pi} W(\phi) \sin (j+1) \phi \sin (k+1) \phi d \phi, j, k=0,1,2, \cdots$. Then $\boldsymbol{T}-\boldsymbol{S}=\boldsymbol{H}$.

$$
\begin{array}{cl}
\text { Proof. } & w_{j-k}-s_{j, k}=\frac{1}{\pi} \int_{0}^{\pi} W(\phi) \cos (j-k) \phi d \phi \\
& -\frac{2}{\pi} \int_{0}^{\pi} W(\phi) \sin (j+1) \phi \sin (k+1) \phi d \phi . \\
& =\frac{1}{\pi} \int_{0}^{\pi} W(\phi) \cos (j+k+2) d \phi .
\end{array}
$$

We can now state a specialization of Kato's theorem in a form suitable for our application. It is understood that in the statement $\boldsymbol{T}$ and $\boldsymbol{S}$ need not necessarily be the operators we have already defined.

Theorem 5. (Kato). Suppose $\boldsymbol{T}$ and $\boldsymbol{S}$ are self-adjoint operators on a separable Hilbert space $\mathscr{L}$ such that $\boldsymbol{T}-\boldsymbol{S}=\boldsymbol{H} \in \mathrm{TC}$ and $\boldsymbol{T}$ is AC. Let $\gamma$ and $\boldsymbol{E}(\cdot)=\boldsymbol{E}_{\mathrm{AC}}(\cdot)+\boldsymbol{E}_{S}(\cdot)$ be the Borel set and decomposition respectively guaranteed by Lemma 3 . Then

(i) as $t \rightarrow \infty$, exp (itT) exp (-itS) $\boldsymbol{E}(\gamma)$ converges strongly to an isometric mapping $\boldsymbol{U}$ of $\boldsymbol{E}(\gamma)$ L onto $\mathscr{L}$.

(ii) $\boldsymbol{U}^{-1}$ is the strong limit as $t \rightarrow \infty$ of $\exp (i t \boldsymbol{S}) \exp (-i t \boldsymbol{T})$.

(iii) The self-adjoint operator $\boldsymbol{S}_{\mathrm{AC}}=\boldsymbol{E}(\gamma) \boldsymbol{S E}(\gamma)$ on $\boldsymbol{E}(\gamma) \mathscr{L}$ is unitarily equivalent to $\boldsymbol{T}$, with $I=\boldsymbol{U} \boldsymbol{S}_{\mathrm{AC}} \boldsymbol{U}^{-1}$.

From this follows the following spectral analysis theorem for $\boldsymbol{T}$.

Theorem 6. Suppose $W$ is a real even AC function on $(-\pi, \pi)$ whose derivative $W^{\prime}$ has an absolutely convergent Fourier series. Then 
the Toeplitz operator $\boldsymbol{T}(W(\phi))$ is unitarily equivalent to to the multiplication operator $\boldsymbol{M}_{\mathrm{AC}}: f \rightarrow W \cdot f$ on $L^{2}(A)$ (see 4.3).

Proof. The hypotheses of Theorem 5 are satisfied via Lemma 4, the discussion following Lemma 3, and Theorem 3. Thus $\boldsymbol{T}$ is unitarily equivalent to $S_{A C}$. Since $\left\{f_{n}\right\}_{0}^{\infty} \rightarrow 2^{1 / 2} \sum_{n=0}^{\infty} f_{n} \sin (n+1) \phi$ is an isometry of $l^{2}$ onto $L^{2}(0, \pi)$, it follows that $\boldsymbol{S}_{\mathrm{AC}}$ is unitarily equivalent to $\boldsymbol{M}_{\mathrm{AC}}$. Thus $\boldsymbol{T}$ is unitarily equivalent to $\boldsymbol{M}_{\mathrm{AC}}$.

COROLlaRy 1. Suppose $W(\phi)=w_{0}+2 \sum_{1}^{m} w_{n} \cos n \phi$, where the $w_{n}$ are real and $m$ is a positive integer. Then $\boldsymbol{T}(W(\phi))$ is unitarily equivalent to the multiplication operator $\boldsymbol{M}: f \rightarrow W \cdot f$ on $L^{2}(0, \pi)$.

Proof. In this case $\boldsymbol{M}=\boldsymbol{M}_{\mathrm{AC}}$. (See Putnam [11], p. 522). Now use Theorem 6.

If $W^{\prime}$ is $\mathrm{AC}$ and $W^{\prime \prime} \in L^{2}(0, \pi)$ then $\Sigma_{n}\left|w_{n}\right|<\infty$. Hence a $W$ satisfying Theorem 6 can haye intervals of constancy. If such is the case, then $\boldsymbol{M}$ has an infinite number of eigenvectors. Thus one cannot validly replace " $\boldsymbol{M}_{\mathrm{AC}}$ " and " $L^{2}(A)$ " by " $\boldsymbol{M}$ " and " $L^{2}(0, \pi)$ " respectively in the statement of Theorem 6 , since $T$ has no point spectra.

We can easily deduce 1.2 from Corollary 1. $\boldsymbol{T}(W(2 \cos n \phi))$ is unitarily equivalent to multiplication by $2 \cos n \phi$ on $L^{2}(0, \pi), n=1,2, \cdots$, and hence to $2 \cos \left(n \operatorname{arc} \cos \frac{1}{2} \boldsymbol{T}(2 \cos \phi)\right)=2 T_{n}\left(\frac{1}{2} \boldsymbol{T}(2 \cos \phi)\right)$ on $l^{2}$.

It would be of great interest to evaluate the limits in Theorem 5 (ii) and (iii) so one could exhibit the unitary transformation of Theorem 6. One could then have a super-abundance of new unitary operators. We pose this as an unsolved problem.

5. Appendix. C. R. Putnam has extended the theory he set forth in [11] in his recent article "On Toeplitz matrices, absolute continuity and unitary equivalence", Pacific J. Math., 9 (1959), 837-846. He proves that $\boldsymbol{T}$ is AC provided that $\boldsymbol{A}_{0}$ is bounded and $\boldsymbol{M}=\boldsymbol{M}_{\mathrm{AC}}$. whence, using [13], he proves Theorem 6 under the added hypothesis that $\boldsymbol{M}=\boldsymbol{M}_{\mathrm{AC}}$.

It is interesting to compare our proof that $\boldsymbol{T}$ is AC with Putnam's weaker version of that result. He applies his abstract theory of commutators, while we exhibit the resolvent of $\boldsymbol{T}$ and employ the rather deep function-theoretic results of [1].

\section{REFERENCES}

1. N. Aronszajn and W. F. Donoghue, Jr., On exponential representations of analyticfunctions in the upper halfplane with positive imaginary part, Journal D'Analyse Mathématique, 5 (1956-57), 321-388.

2. A. Calderon, F. Spitzer, and H. Widom, Inversion of Toeplitz matrices. Cornell University contract report, March, 1958.

3. Ulf Grenander and Gabor Szegö, Toeplitz forms and their applications, University of 
California, 1958.

4. Paul R. Halmos, Introdution to Hilbert space and the theory of spectral multiplicity, New York, 1951.

5. P. Hartman and A. Wintner, On the spectra of Toeplitz's matrices, Amer. J. Math., 72 (1950), 359-366.

6. - The spectra of Toeplitz's matrices Amer. J. Math. 76 (1954), 867-882.

7. Tosio Kato, On finite-dimensional perturbations of selfadjoint operators, Journal Math Soc. Japan, 9 (1957), 239-249.

8. - Perturbation of continuous spectra by trace class operators, Proc. Japan Acad., 33 (1957), 260-264.

9. Shige Toshi Kuroda, On the existence and the unitary property of the scattering operator, mimeographed notes from Dept. of Physics, Univ. of Tokyo, Jan., 1959.

10. - Perturbation of continuous spectra by unbounded operators, to be published. 11. C. R. Putnam, Commutators and absolutely continuous operators, Trans. Amer. Math. Soc., 87 (1958). 513-525.

12. F. Riesz, and B. Sz.-Nagy, Leçons d'analyse fonctionelle, Budapest, 1952.

13. M. Rosenblum, Perturbation of the continuous spectrum and unitary equivalence Pacific J. Math., 7 (1957), 997-1010.

14. S. Saks, Theory of the integral, Warszawa-Lwow, 1937.

UNIVERSITY OF VIRGINIA 


\section{PACIFIC JOURNAL OF MATHEMATICS}

\section{EDITORS}

\author{
David Gilbarg \\ Stanford University \\ Stanford, California \\ F. H. Brownell \\ University of Washington \\ Seattle 5 , Washington
}

\author{
A. L. Whiteman \\ University of Southern California \\ Los Angeles 7, California \\ L. J. PAIGE \\ University of California \\ Los Angeles 24, California
}

\section{ASSOCIATE EDITORS}

\author{
E. F. BECKENBACH \\ T. M. CHERRY \\ D. DERRY
}
E. HEWITT
A. HORN
L. NACHBIN
M. OHTSUKA
H. L. ROYDEN
M. M. SCHIFFER

E. SPANIER

E. G. STRAUS

F. WOLF

\section{SUPPORTING INSTITUTIONS}

\author{
UNIVERSITY OF BRITISH COLUMBIA \\ CALIFORNIA INSTITUTE OF TECHNOLOGY \\ UNIVERSITY OF CALIFORNIA \\ MONTANA STATE UNIVERSITY \\ UNIVERSITY OF NEVADA \\ NEW MEXICO STATE UNIVERSITY \\ OREGON STATE COLLEGE \\ UNIVERSITY OF OREGON \\ OSAKA UNIVERSITY \\ UNIVERSITY OF SOUTHERN CALIFORNIA
}

\author{
STANFORD UNIVERSITY \\ UNIVERSITY OF TOKYO \\ UNIVERSITY OF UTAH \\ WASHINGTON STATE COLLEGE \\ UNIVERSITY OF WASHINGTON

AMERICAN MATHEMATICAL SOCIETY
CALIFORNIA RESEARCH CORPORATION
HUGHES AIRCRAFT COMPANY
SPACE TECHNOLOGY LABORATORIES
NAVAL ORDNANCE TEST STATION

Mathematical papers intended for publication in the Pacific Journal of Mathematics should be typewritten (double spaced), and the author should keep a complete copy. Manuscripts may be sent to any one of the four editors. All other communications to the editors should be addressed to the managing editor, L. J. Paige at the University of California, Los Angeles 24, California. ..

50 reprints per author of each article are furnished free of charge; additional copies may be obtained at cost in multiples of 50 .

The Pacific Journal of Mathematics is published quarterly, in March, June, September, and December. The price per volume (4 numbers) is $\$ 12.00$; single issues, $\$ 3.50$. Back numbers are available. Special price to individual faculty members of supporting institutions and to individual members of the American Mathematical Society: $\$ 4.00$ per volume; single issues, $\$ 1.25$.

Subscriptions, orders for back numbers, and changes of address should be sent to Pacific Journal of Mathematics, 2120 Oxford Street, Berkeley 4, California.

Printed at Kokusai Bunken Insatsusha (International Academic Printing Co., Ltd.), No. $6_{4}$ 2-chome, Fujimi-cho, Chiyoda-ku, Tokyo, Japan.

PUBLISHED BY PACIFIC JOURNAL OF MATHEMATICS, A NON-PROFIT CORPORATION

The Supporting Institutions listed above contribute to the cost of publication of this Journăl, but they are not owners or publishers and have no responsibility for its content or policies. 


\section{Pacific Journal of Mathematics}

\section{Vol. 10, No. $3 \quad$ November, 1960}

Glen Earl Baxter, An analytic problem whose solution follows from a simple

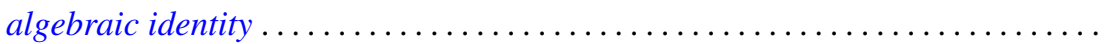

Leonard D. Berkovitz and Melvin Dresher, A multimove infinite game with linear payoff. .

Earl Robert Berkson, Sequel to a paper of A. E. Taylor ......................

Gerald Berman and Robert Jerome Silverman, Embedding of algebraic systems.... 767

Peter Crawley, Lattices whose congruences form a boolean algebra . . . . . ...... 777

Robert E. Edwards, Integral bases in inductive limit spaces . . . . . . . . . . . . . . .

Daniel T. Finkbeiner, II, Irreducible congruence relations on lattices . . . . . . . . . .

William James Firey, Isoperimetric ratios of Reuleaux polygons . . . . . . . . . . . 787

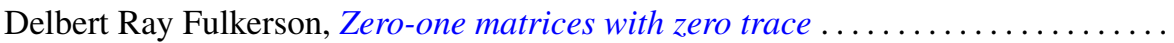

Leon W. Green, A sphere characterization related to Blaschke's conjecture........

Israel (Yitzchak) Nathan Herstein and Erwin Kleinfeld, Lie mappings in

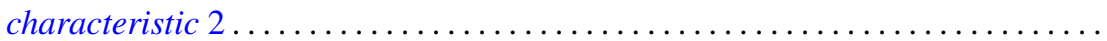

Charles Ray Hobby, A characteristic subgroup of a $p$-group .................

R. K. Juberg, On the Dirichlet problem for certain higher order parabolic

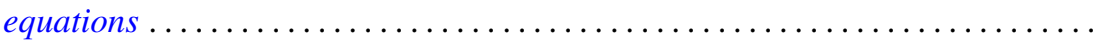

Melvin Katz, Infinitely repeatable games ......................

Emma Lehmer, On Jacobi functions . . . . . . . . . . . . . . . . . . . . . . . . .

D. H. Lehmer, Power character matrices

Henry B. Mann, A refinement of the fundamental theorem on the density of the sum of two sets of integers.

Marvin David Marcus and Roy Westwick, Linear maps on skew symmetric matrices: the invariance of elementary symmetric functions . .

Richard Dean Mayer and Richard Scott Pierce, Boolean algebras with ordered

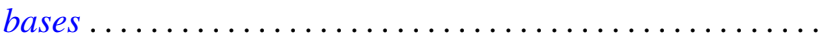

Trevor James McMinn, On the line segments of a convex surface in $E_{3} \ldots$

Frank Albert Raymond, The end point compactification of manifolds ..

Edgar Reich and S. E. Warschawski, On canonical conformal maps of regions of arbitrary connectivity

Marvin Rosenblum, The absolute continuity of Toeplitz's matrices...

Lee Albert Rubel, Maximal means and Tauberian theorems . .

Helmut Heinrich Schaefer, Some spectral properties of positive linear operators

Jeremiah Milton Stark, Minimum problems in the theory of pseudo-conformal transformations and their application to estimation of the curvature of the invariant metric.

Robert Steinberg, The simplicity of certain groups ...

Hisahiro Tamano, On paracompactness. .

Angus E. Taylor, Mittag-Leffler expansions and spectral theory .

Marion Franklin Tinsley, Permanents of cyclic matrices ...... . 